\title{
It takes two to bleed: anticoagulation intensity and the host's vascular susceptibility
}

\author{
Benjamin Seeliger ${ }^{1} \mathbb{0}$, Pedro David Wendel-Garcia ${ }^{2}$, Klaus Stahl ${ }^{3}$, Christian Bode $^{4}$ and Sascha David ${ }^{*}$
}

(c) 2022 Springer-Verlag GmbH Germany, part of Springer Nature

We have read with interest the report of ELSO-registry data published by Nunez and coworkers in Intensive Care Medicine analyzing risk factors for bleeding and thrombotic events (BTE) on venovenous extra-corporeal membrane oxygenation (vv-ECMO) and clinical outcomes [1]. The abundance of data derivable from the ELSO-registry allows for powerful modeling, on which we congratulate the authors. Yet, we noticed with, admittedly, some disappointment that the data failed to unveil a single modifiable risk factor. The authors credit the observed decrease of BTE from 2010 to 2017 to advances in circuit composition, cannulation techniques and improved training, yet did not address the elephant in the room, the clinicians' own contribution to tragedy: choice and intensity of anticoagulation.

The problem here is the general lack of consensus regarding all levels of anticoagulation, even on national levels, given the vast room for interpretation offered by the ELSO guidelines: the choice of "intensity" of anticoagulation, its definition and the appropriate means for monitoring, all having considerable consequences on BTE risk.

Our group demonstrated that different heparin monitoring and dosing strategies result in ample differences in BTE occurrence with mean heparin doses differing by $60 \%$ between experienced centers and, quite unsurprisingly, bleeding occurring far more often with higher heparin doses, while thrombotic events peak with lower doses [2].

Besides this iatrogenic risk factor, patient-specific characteristics most likely additionally contribute to

\footnotetext{
*Correspondence: sascha.david@usz.ch

${ }^{2}$ Institute of Intensive Care Medicine, University Hospital Zurich, Zurich, Switzerland
}

Full author information is available at the end of the article susceptibility for BTE. We were surprised that Nunez and coworkers did not consider the underlying etiology for respiratory failure in their main BTE models. This is nothing less than astonishing given that for the last 2 years, we have been confronted with an etiology for respiratory failure, often requiring ECMO support, that carries a tenacious risk for BTEs-coronavirus disease 2019 (COVID-19). Initial reports together with bedside observations of increased risk for thrombotic complications have led intensivists around the globe to ardently anticoagulate at the high end of the usual spectrum [3]. We now know that COVID-19-specific endothelialitis [4] not only drives thrombosis but translates to an up to sixfold increased risk for intracranial hemorrhage on ECMO support with a demoralizing mortality of up to $90 \%$ [5]. It does not seem far-fetched to hypothesize that other etiologies of respiratory failure also present similar phenotypic differences in their response to anticoagulation. Indeed, the authors hint in a supplemental subgroup analysis toward increased bleeding risk with major surgical trauma, but we think that the interaction of anticoagulation with other primary acute respiratory distress syndrome (ARDS) etiologies will more likely yield developable hypotheses.

We agree with the authors that BTE events on ECMO support need to be reduced by individualized approaches. We suggest that identifying and stratifying patients at risk for BTE will facilitate this to maintain the right balance between prothrombotic and pro-hemorrhagic risk.

Since anticoagulation is highly adaptive and thus unattractive to capture within registries, multinational efforts to collect more granular data would be welcomed. Finally, 2 years into the COVID-19 pandemic, with no conceivable end and new SARS-CoV-2 variants in sight, we feel that prospective trials to establish best anticoagulation strategies on ECMO are long overdue.

\section{Springer}




\section{Author details}

${ }^{1}$ Hannover Medical School, Department of Respiratory Medicine and Biomedical Research in End-Stage and Obstructive Lung Disease Hannover, German Center for Lung Research, Hannover, Germany. ${ }^{2}$ Institute of Intensive Care Medicine, University Hospital Zurich, Zurich, Switzerland. ${ }^{3}$ Department of Gastroenterology, Hepatology and Endocrinology, Hannover Medical School, Hannover, Germany. ${ }^{4}$ Department of Anesthesiology and Intensive Care Medicine, University Hospital Bonn, Bonn, Germany.

\section{Declarations}

\section{Conflicts of interest}

The authors declare no conflicts of interest.

\section{Human and animal rights}

This manuscript does not include any data on human or animal subjects.

\section{Publisher's Note}

Springer Nature remains neutral with regard to jurisdictional claims in published maps and institutional affiliations.

Accepted: 5 February 2022

Published: 10 February 2022

\section{References}

1. Nunez Jl, Gosling AF, O'gara B, Kennedy KF, Rycus P, Abrams D et al (2021) Bleeding and thrombotic events in adults supported with venovenous extracorporeal membrane oxygenation: an ELSO registry analysis. Intensive Care Med. https://doi.org/10.1007/S00134-021-06593-X

2. Seeliger B, Döbler M, Friedrich R, Stahl K, Kühn C, Bauersachs J et al (2021) Comparison of anticoagulation strategies for veno-venous ECMO support in acute respiratory failure. Crit Care 24:701. https://doi.org/10.1186/ S13054-020-03348-W

3. Doyle AJ, Hunt BJ, Sanderson B, Zhang J, Mak SM, Benedetti G et al (2021) A comparison of thrombosis and hemorrhage rates in patients with severe respiratory failure due to coronavirus disease 2019 and influenza requiring extracorporeal membrane oxygenation. Crit Care Med 49:E663E672. https://doi.org/10.1097/Ccm.0000000000004971

4. Ackermann M, Verleden SE, Kuehnel M, Haverich A, Welte T, Laenger F et al (2020) Pulmonary vascular endothelialitis, thrombosis, and angiogenesis in Covid-19. N Engl J Med 383:120-128. https://doi.org/10.1056/ Nejmoa2015432

5. Seeliger B, Döbler M, Hofmaenner DA, Garcia-Wendel PD, Schuepbach RA, Schmidt JJ et al (2021) Intracranial hemorrhages on extracorporeal membrane oxygenation: differences between coronavirus disease 2019 and other viral acute respiratory distress syndrome. Crit Care Med. https://doi.org/10.1097/CCM.0000000000005441 